\title{
Modelagem da Produtividade e Gestão da Qualidade Acadêmica
}

\author{
Annibal Parracho Sant'Anna \\ Dpto. Eng. Produção - EE - UFF \\ Rua Passo da Pátria, 156 \\ 24210-240 Niterói-RJ - Brasil
}

\section{Resumo}

Apresenta-se aqui um modelo hierarquizado e dinâmico para a produçāo dos departamentos de uma universidade. O modelo é hierarquizado porque os coeficientes técnicos de utilizaçāo da força de trabalho docente sāo modelados em um segundo nível; podese, com isto, acompanhar as variaçōes destes coeficientes, de departamento para departamento. E é dinâmico no sentido em que os mesmos coeficientes variam a longo do tempo. Além disto, a verificaçāo do ajustamento do modelo à realidade e a seleção de observaçōes aberrantes sāo usadas para suscitar mudanças automáticas na especificaçāo do modelo a ser ajustado adiante, sem prejuízo da continuidade da avaliaçāo.

\section{Abstract}

This paper presents a dynamic hierarchical model for the production of university departments. It is a hierarchical model because technical coefficients of the academic production function are modeled as function of second level explanatory variables in such a way that such production function is allowed to change coherently from one department to another. It is dynamic in the sense that these technical coefficients are also allowed to change through time. Moreover, validation and outlier selection techniques are applied to feed an automatic model revision procedure that preserves continuity in the evaluation process.

Palavras Chaves:

Gestão da Qualidade, Avaliaçāo Acadêmica, Estimaçāo Recursiva, Modelos Hierárquicos.

Keywords:

Hierarchical Models, Quality Management, Academic Evaluation, Recursive Estimation.

\section{Introdução}

A complexidade da produção universitária, a importância da liberdade de investigação e da preservação da autoridade docente exigem a autonomia departamental. Além disso, ainda que dependa também das decisões centrais sobre a organização das atividades, a disponibilidade de recursos materiais, etc., é a atuação direta dos docentes nos departamentos que determina a qualidade dos resultados acadêmicos. Por isso, é a nível de departamento que se tem de realizar o maior esforço para a promoção da qualidade na Universidade. Em especial, a virtude mais importante em qualquer sistema de avaliação do desempenho acadêmico é o seu poder de chamar a atenção dos professores para as possibilidades de melhoria da qualidade e de fornecer informação que oriente o seu esforço nessa direção.

O sistema de avaliação aqui proposto visa a prover os departamentos de informação objetiva e estimular a participação de seus membros no esforço de elevação da qualidade acadêmica. Fundamentase no reconhecimento da complexidade e da diversidade do processo de produção universitário. Não pretende identificar nenhuma forma ideal de organização válida para todos os departamentos, mas procura medir as diferenças em termos que permi- 
tam explicar o efeito de métodos e procedimentos sobre o processo e a qualidade de seus resultados.

Para isto, se baseia em um modelo para a utilização do recurso docente na produção dos grandes agregados de pesquisa, ensino e extensão. A estimação de coeficientes de produção de pesquisa, ensino e extensão individualizadamente não exige que se dissociem esses aspectos da atividade acadêmica. Para explicitar essas interações, o modelo, em um segundo nível, explica a variação desses coeficientes ao longo dos departamentos em função de variáveis explicativas próprias.

A fundamentação na modelagem da produção ácadêmica dá ao sistema duas características principais. A primeira é que fornece a cada departamento informação sobre o seu desempenho não apenas em termos globais e comparativos com os de outros departamentos, mas, também, em termos de valores para os parâmetros da função de produção próprios do departamento. Com isto, ele pode identificar pontos em que deva concentrar esforços para a elevação da qualidade.

A segunda característica é que o resultado final de cada rodada de avaliação é a seleção de um pequeno grupo de departamentos cujo desempenho mais se afasta do previsto pelo modelo. Nestes departamentos se concentra a análise, buscando identificar aspectos específicos da atividade neles desenvolvida que o modelo não esteja considerando devidamente.

Ao permitir a evolução do modelo ao longo do tempo, através da revisão periódica da composição de suas variáveis explicativas e através da estimação iterativa dos seus parâmetros, abre-se espaço para o seu aperfeiçoamento. É claro que não se pretende chegar a representar nunca, por este modelo, o verdadeiro mecanismo da atividade acadêmica em nenhum departamento real. Pretende-se, entretanto, produzir aproximações que instiguem a refletir sobre esse processo e, em especial, detectar pontos onde essa reflexão possa ser mais produtiva. Ao selecionar para análise os departamentos em que é maior a distância entre os resultados observados e a expectativa gerada pelo modelo, se maximiza a possibilidade de que esta análise venha a contribuir para o aperfeiçoamento do modelo.

O sistema coloca à disposição dos departamentos, cada ano, um amplo levantamento de dados, estimativas de coeficientes técnicos de produção de pesquisa, ensino e extensão, individualizados por departamento, assim como uma medida global da produção de cada um. Pede em troca, a cada um, sugestões quanto a parâmetros que orientem a composição de medidas de produção adequadas para a respectiva área de conhecimento. Pede, também, que estejam preparados para apresentar, caso selecionados, sugestões de novos indicadores da utilização dos recursos, tanto em termos de produtividade quanto de qualidade.

Deste modo, se rompe o dilema quantidade $\mathrm{X}$ qualidade, objetividade X subjetividade. Obtemos medidas objetivas de quantidades que são sempre aproximações para as qualidades que gostaríamos de avaliar. Em contrapartida, a divulgação destas medidas suscita automaticamente uma reflexão que ajuda a compreendê-las mais corretamente.

A Seção seguinte contém uma descrição das linhas básicas do modelo hierarquizado dinâmico utilizado para representar a produção acadêmica. É seguida pela explicitação de seus mecanismos de revisão periódica. Na Seção 4 , é apresentado o modelo ajustado à produção dos departamentos da Universidade Estadual Paulista Júlio Mesquita Filho (UNESP).

\section{O Modelo Hierarquizado para a Produção}

O modelo aqui ajustado é um modelo hierarquizado dinâmico (ver Gamerman e Migon, 
1993) baseado em uma aproximação para a utilização do recurso docente em suas diversas atribuições na Universidade. O princípio do seu emprego em um sistema de avaliação de departamentos é o mesmo da Data Envelopment Analysis (ver Banker e Morey, 1986 e Sinuany-Stern et alii, 1993). A diferença está em que, em vez de enfatizar a comparação do desempenho de cada unidade de observação com o desempenho ótimo em uma unidade composta com o mesmo padrão de utilização do recurso, a estimação prévia dos parâmetros do modelo transfere a ênfase para a comparação com o desempenho médio anteriormente observado na própria unidade de observação.

A unidade de observação é a unidade básica de gerência na Universidade, o departamento. O modelo adota para todos os departamentos a mesma especificação, separando a produção em três grandes blocos: Pesquisa, Ensino e Extensão. Os departamentos estão divididos em oito grupos acompanhando as oito grandes áreas do conhecimento: de Ciências Biológicas Básicas, de Ciências Agrícolas e Veterinárias, de Ciências da Saúde, de Ciências Exatas e da Natureza, de Engenharias, de Ciências Humanas, de Ciências Sociais Aplicadas e de Lingüística, Letras e Artes. Estes grupos podem ser subdivididos, levando-se em conta características como, por exemplo, as diferentes possibilidades de interação externa devidas à localização do departamento em unidade típica da respectiva área do conhecimento ou não.

Além de variar entre as áreas do conhecimento, admite-se que os coeficientes da função de produção modelada variem dentro de cada grupo, em resposta a características do processo de produção próprio e, também, da maior ou menor utilização da sua força de trabalho docente em tarefas de administração acadêmica e do maior ou menor comprometimento dessa força de trabalho com complementação da sua formação profissional.
Cada variável explicativa é composta combinando informações numéricas disponíveis. Assim, no primeiro nível, o resultado da atividade de ensino é medido por uma combinação linear de indicadores do volume do ensino em sala de aula, como o somatório dos créditos cursados por cada aluno, com medidas de orientação de trabalhos, como projetos finais e teses defendidas pelos alunos. Do mesmo modo, o resultado da atividade de pesquisa é medido combinando números de artigos publicados e de projetos de pesquisa em andamento e o resultado nas atividades de extensão combinando o volume de serviços de cuja execução o Departamento participa no momento com números de atendimentos diretos prestados.

Essas medidas numéricas são aproximações que podem ser sempre aperfeiçoadas. Da simples contagem de créditos em disciplinas dos alunos, pode-se passar para somas de créditos ponderados em que cada crédito é valorizado, por exemplo, pelo resultado da verificação final da aprendizagem ou pela avaliação final da qualidade do ensino recebido, obtida do aluno. Os artigos de pesquisa podem ser valorizados pela reputação dos veículos em que são divulgados ou pela relevância que lhe atribuam especialistas ad hoc. E assim por diante.

Para compor as três variáveis indicativas do volume da produção, as medidas numéricas relevantes são combinadas com pesos que variam de uma área de conhecimento para outra. Estes pesos são obtidos de especialistas representando os departamentos da área, mediante aplicação de técnicas de quantificação de preferências, cujos resultados são a seguir revistos pelos especialistas. Um importante elemento de motivação para a qualidade embutido no sistema é o permanente envolvimento dos professores no processo de revisão desses pesos.

Processo análogo aplica-se na construção das variáveis explicativas do segundo nível. Dezenas de indicadores da complexidade do processo de pro- 
PRODUÇÃO

dução e do desvio da força de trabalho docente para a complementação da sua formação profissional e para as atividades de administração acadêmica são combinadas, com pesos obtidos dos especialistas da área, em um processo de revisões sucessivas.

Igualmente aproximada é a variável dependente: a soma das remunerações dos docentes do departamento no período é usada como medida aproximada da força de tr...balho docente envolvida no processo produtivo. Ao contrário do que ocorre com as variáveis explicativas, aqui os pesos são rígidos: o peso de cada docente é a sua remuneração. Deixando fixo o mecanismo de construção da variável dependente e concedendo maior flexibilidade apenas à construção das variáveis explicativas, facilitamos a comparação das predições oferecidas ao longo do tempo pelo ajustamento do modelo.

Temos, assim, simplificadamente, a seguinte função de produção com coeficientes variáveis.

$$
\begin{aligned}
R_{i} & =b_{1 i} E_{i}+b_{2 i} P_{i}+b_{3 i} X_{i}+u_{1 i}, \\
b_{j i i} & =c_{1 j i}+c_{2 j i j} A_{i}+c_{3 j i} B_{j i}+u_{2 j i}, \\
\text { para j } & =1,2 \text { e } 3,
\end{aligned}
$$

Na representação acima, $i$ variando ao longo do conjunto de departamentos da Universidade, $\mathrm{R}_{\mathrm{i}}$ denota o custo de utilização do recurso docente, $E_{i}$, $\mathrm{P}_{\mathrm{i}}$ e $\mathrm{X}_{\mathrm{i}}$ denotam, respectivamente, o volume de produção de ensino, pesquisa e extensão, $\mathrm{A}_{\mathrm{i}}$ denota $o$ volume do envolvimento dos docentes em atividades de complementação da própria formação e em funções de administração acadêmica e $B_{\mathrm{ji}}$ o volume de atividades intermediárias registradas no processo de produção do produto (Ensino, Pesquisa ou Extensão) do qual $b_{\mathrm{ji}}$ representa o coeficiente técnico de utilização do fator trabalho docente. Finalmente, un denota perturbação estocástica de nível n.

Os coeficientes do modelo, específicos de cada departamento, são estimados recursivamente, ao longo do tempo, a partir de estimativas iniciais idênticas ao longo dos departamentos da mesma área do conhecimento e obtidas, também, da consul- ta a especialistas. Estes especialistas podem ser orientados no sentido de fornecerem como estimativas iniciais avaliações não dos coeficientes técnicos realmente encontrados na instituição, mas daqueles desejados ou considerados ideais para a mesma. Uma consequiência de seguir esta última orientação é encontrarmos, nos primeiros anos de utilização do modelo, um pior ajustamento aos dados e, pelo critério de maiores erros de predição, selecionarmos, nesses primeiros ajustamentos, os departamentos cujo desempenho mais se afasta, não do desempenho médio observado, mas do desempenho médio desejado.

Cada vez que um novo conjunto de dados é colhido, as estimativas são revistas. $O$ processo de revisão se inicia com uma fase descendente, em que, primeiro, as estimativas dos coeficientes da função de produção são atualizadas; depois, estas estimativas dos coeficientes do primeiro nível são usadas para atualizar as estimativas dos coeficientes do segundo nível e assim por diante. E se completa com uma fase ascendente, de suavização, em que, primeiro, a equação do último nível com os coeficientes recém-atualizados é usada para suavizar as estimativas dos coeficientes do penúltimo nível; depois, estas são usadas para suavizar as dos coeficientes do nível imediatamente anterior; e assim por diante, até chegar ao primeiro nível.

No caso de apenas dois níveis, o algoritmo iterativo pode ser representado formalmente pelas duas etapas descritas abaixo.

Etapa de atualização:

$\mathrm{b}_{\mathrm{jit}}{ }^{*}=\mathrm{b}_{\mathrm{jit}}-1+\mathrm{f}_{\mathrm{j}}\left(\mathrm{R}_{\mathrm{it}}-\left(\mathrm{b}_{1 \mathrm{it}}-1 \mathrm{E}_{\mathrm{it}}+\mathrm{b}_{2 \mathrm{it}}-1 \mathrm{P}_{\mathrm{it}}+\right.\right.$ $\left.\left.b_{3 i t}-1 X_{i t}\right)\right)$, para $j=1,2$ e 3,

$\mathrm{c}_{\mathrm{kj \textrm {jit }}}=\mathrm{c}_{\mathrm{kj \textrm {j } t - 1}}+\mathrm{g}_{\mathrm{kj}}\left(\mathrm{b}_{\mathrm{jit}} *-\left(\mathrm{c}_{\mathrm{j} \mathrm{jit}-1}+\mathrm{c}_{2 \mathrm{jit}-1} \mathrm{~A}_{\mathrm{it}}+\right.\right.$ $\left.\left.\mathrm{c}_{3 \mathrm{jit}-1} \mathrm{~B}_{\mathrm{jit}}\right)\right)$, para j e $\mathrm{k}=1,2$ e 3 .

Aqui, $b_{\mathrm{jit}}{ }^{*}$ e $b_{\mathrm{jit}-1}$ denotam, respectivamente, a estimativa atualizada e a estimativa final do ano anterior para $b_{\mathrm{ji}}$. Analogamente, $\mathrm{c}_{\mathrm{kj \textrm {j }}} \mathrm{e} \mathrm{c}_{\mathrm{kj \textrm {jit } - \mathrm { l }}}$ deno- 
tam, respectivamente, a estimativa atualizada e a estimativa final do ano anterior para $\mathrm{c}_{\mathrm{kji}}$; o detalhe importante é que as estimativas para as $\mathrm{c}_{\mathrm{kji}}$ no ano $\mathrm{t}$ são construídas usando as estimativas para $b_{\mathrm{ji}}$ no mesmo ano, indicadas na linha superior por $b_{j i t} * \cdot f_{j} e$ $\mathrm{g}_{\mathrm{kj}}$ são funções crescentes tendo como argumento os erros de predição indicados. Uma alternativa para a construção destas funções é usar o fator de correção do algoritmo de mínimos quadrados recursivos.

Etapa de suavização:

para $\mathrm{j}=1,2$ e 3 .

$$
\mathrm{b}_{\mathrm{jit}}=\mathrm{c}_{1 \mathrm{jit}}+\mathrm{c}_{2 \mathrm{jit}} \mathrm{A}_{\mathrm{it}}+\mathrm{c}_{3 \mathrm{jit}} \mathrm{B}_{\mathrm{jit}},
$$

Aqui, as $c_{\text {kjit }}$ são as estimativas obtidas no final da etapa de atualização. Uma vez obtidas, estas $\mathrm{c}_{\mathrm{kjit}}$ finais são utilizadas para produzir as $b_{j i t}$, estimativas finais do ano t para os coeficientes do primeiro nível.

Ao final de cada iteração, os erros de predição do primeiro nível, dados por $\mathrm{R}_{\mathrm{it}}-\left(\mathrm{b}_{1 \mathrm{it}} \mathrm{E}_{\mathrm{it}}+\mathrm{b}_{2 \mathrm{it}}\right.$ $\left.\mathrm{P}_{\mathrm{it}}+\mathrm{b}_{3 \mathrm{it}} \mathrm{X}_{\mathrm{it}}\right)$, são padronizados em função da variância observada no grupo de departamentos da mesma área. Os departamentos com maiores erros de predição padronizados são selecionados para uma análise, cujo resultado apoia a próxima revisão do modelo.

\section{Verificação do Modelo}

A constituição dos grupos e a composição das variáveis representativas da produção agregada em pesquisa, ensino e extensão, assim como do comprometimento com a própria formação profissional e com a administração acadêmica, deve ser decidida através de um amplo processo de consulta aos departamentos. A cada iteração, essa decisão deve ser revista. A finalidade principal disto é estimular o envolvimento na gestão da qualidade.

Por outro lado, subsídios para a revisão são fornecidos, não apenas pelo resultado da análise em profundidade dos departamentos selecionados, mas, também, pela aplicação de técnicas de Análise
Estatística Multivariada aos dados brutos para detectar defeitos na especificação ajustada, tais como alta correlação entre variáveis explicativas ou baixa correlação entre estas e a variável que pretendem explicar.

Na análise dos departamentos com valores extremos, a revisão do modelo é orientada por algumas diretrizes básicas, que se impõem naturalmente. De um lado, produtos podem estar sendo priorizados indevidamente, características do processo de produção de determinado grupo de departamentos podem estar sendo desprezadas, etc. De outro lado, altos erros de predição podem estar associados ao uso de instrumentos de gestão que não se cogita de quantificar. Assim, a análise dos departamentos escolhidos deverá visar a dois objetivos distintos, quais sejam: a) aprofundamento da avaliação da atividade acadêmica, contemplando, pelo menos, a verificação da adequação dos indicadores coletados e dos pesos usados na composição das variáveis, correção das medidas registradas e correção da classificação em áreas; b) avaliação dos procedimentos de administração da qualidade empregados, considerando, aspectos como: focalização no cliente próximo (aluno) e remoto (sociedade), compromisso da liderança, uso de instrumentos de planejamento, documentação dos processos acadêmicos, qualificação do corpo docente e dos recursos humanos em geral, atenção às interações internas ao processo e uso de procedimentos de avaliação interna dos resultados.

O instrumento básico para a coleta de informações destinadas à revisão do modelo é constituído pelos questionários de realimentação, cuja aplicação acompanha o processo de análise em profundidade dos departamentos selecionados. Os questionários de realimentação procuram identificar, sucessivamente: a) objetivos de qualidade da unidade, recursos de que mais depende no momento o desempenho em relação a tais objetivos e relações pelas 


\section{PRODUÇÃO}

quais esta dependência se consubstancia; b) consistência desses objetivos, recursos e relações com aqueles expressos no modelo hierárquico ajustado; c) propostas de inclusões e exclusões de variáveis nos diversos níveis do modelo, de perguntas nos questionários ou de alterações no sistema de pesos com que se compõem as variáveis do modelo.

Assim, os resultados da análise podem ser indicações de correções no modelo ou na sistemática toda de controle, tanto quanto nos outros componentes da administração acadêmica.

Além da correção de distorções, também acréscimos, para suprir insuficiências da especificação detectadas, podem ser sugeridos. Instrumentos gerenciais decisivos para a qualidade acadêmica como a eficácia do planejamento pedagógico e o desenvolvimento de metodologia de auto-avaliação poderiam ser medidos e não são. A ausência de dados a respeito reflete menos a deficiência do seu levantamento que a do seu uso efetivo. Os departamentos que venham a utilizá-los, à medida em que, por apresentarem resíduos no ajustamento altamente positivos, sejam chamados a explicar seus resultados, deverão estar preparados para propor mecanismos para o adequado registro do uso esses instrumentos.

\section{Implementação na Prática}

Face à dificuldade de determinar a priori as variáveis que medem mais adequadamente a produção acadêmica em cada área do conhecimento, não se deve esperar que o modelo ajustado aos dados colhidos nos primeiros anos reflita com precisão as atividades realizadas. Assim, é de esperar que os maiores erros de predição se verifiquem nos departamentos com valores extremos no volume de recursos docentes observados: a dificuldade geral do modelo de explicar a utilização do recurso crítico será tanto maior quanto maior for a variação no volume do recurso utilizado.

Outra fonte de distorção que se deve esperar encontrar nas implementações iniciais está associada à presença de incorreções nos registros. Esses erros são devidos, principalmente, a imprecisões em critérios de classificação e falhas de padronização em procedimentos de coleta inevitáveis no primeiro momento e corrigidos gradualmente nas sucessivas revisões do modelo.

Tem sido realizadas (Sant'Anna, Souza e Cordeiro, 1995) simulações de ajustamento de especificações alternativas baseadas em mudanças dos pesos atribuídos às variáveis brutas usadas na construção de cada produto agregado. Mudanças orientadas no sentido de aumentar o peso atribuído às variáveis cujos instrumentos de coleta revelam-se menos suscetíveis à influência de erros devidos à interpretação equivocada das instruções de preenchimento resultaram em grande melhora no ajustamento e consideráveis alterações na ordenação dos erros de predição padronizados. Mas, os departamentos selecionados pelo alto valor absoluto dos seus erros de predição não mudam muito.

O ajustamento do modelo utilizado para a produção acadêmica dos departamentos da UNESP permite medir a cada ano, para cada departamento, as seguintes variáveis:

PRODUÇÃO DE ENSINO: indicador de resultado final da produção de Ensino, obtido padronizando o número de disciplinas de graduação e pós-graduação stricto sensu oferecidas e de dissertações, teses e trabalhos finais orientados.

PRODUÇÃO DE PESQUISA: indicador de resultado final da produção de Pesquisa, obtido padronizando o número de publicações de artigos de pesquisa, livros e capítulos de livros e, para os departamentos de Artes, exposições, gravações e recitais correspondentes.

PRODUÇÃO DE EXTENSÃO: indicador de resultado final da produção de Extensão, obtido 
padronizando o número de consultorias, assessorias e projetos parceria.

COEFICIENTE DE PRODUÇÃO DE ENSINO: taxa estimada de utilização do recurso docente nas atividades de Ensino.

COEFICIENTE DE PRODUÇÃO DE PESQUISA: taxa estimada de utilização do recurso docente nas atividades de Pesquisa.

COEFICIENTE DE PRODUÇÃO DE EXTENSÃO: taxa estimada de utilização do recurso docente nas atividades de Extensão.

REMUNERAÇÃO OBSERVADA: afastamento padronizado da folha de pagamento do pessoal docente do departamento da média da área do conhecimento respectiva.

REMUNERAÇÃO PREDITA: afastamento da folha de pagamento do pessoal docente do departamento da média da área do conhecimento respectiva, medida combinando os indicadores de produção com as taxas estimadas de utilização respectivas.

DESVIO PADRONIZADO: diferença entre a REMUNERAÇÃO PREDITA e a REMUNERAÇÃO OBSERVADA, dividida pelo desvio padrão estimado para a área do conhecimento.

\section{Considerações Finais}

O sistema de avaliação baseado em um modelo para a produção acadêmica a dados fornecidos pelos departamentos coloca à disposição dos mesmos informação sobre a sua própria produção que só pode ser bem apreciada em conjunto com a de seus pares. Em contrapartida, suscita uma investigação em profundidade sobre a qualidade dessa produção em departamentos selecionados.

O sistema de avaliação deve ser concebido em caráter permanente. As sucessivas avaliações envolvem, não apenas a coleta de novos dados e o seu ajustamento ao modelo, mas, também, a revisão das especificações das funções de produção incorporadas ao modelo, a partir de uma reflexão estimulada pela análise dos departamentos de desempenho mais surpreendente.

Esta análise permitirá identificar e divulgar a inovação tecnológica que se encontre nos departamentos com desempenho superior ao predito. Fará, também, suscitar inovação nos departamentos que mostrem utilização dos recursos inferior à predita, pelo menos no que concerne ao registro e à documentação dos procedimentos utilizados, etapa básica do processo de garantia da qualidade.

\section{Bibliografia}

Banker, R. D. e Morey, R. C. (1986) Efficiency Analysis for Exogenously Fixed Inputs and Outputs, Operations Research, 34, 513-521.

Gamerman, D. e Migon, H. S. (1993) Dynamic Hierarchical Models, Journal of the Royal Statistical Society B, 55, 629-642.

Sant'Anna, A. P., Souza, A. D. P. e Cordeiro, J. A. (1994) - Modelos Estatísticos na Avaliação Acadêmica, Atas do XI SINAPE, 153-157.

Sinuany-Stern, Z., Mehrez, A. e Barboy, A. (1994) - Academic Departments Efficiency via DEA, Computers Operations Research, 21, 543556. 